\title{
Fast screening of the fatigue properties of thermoplastics reinforced with short carbon fibers based on a heat build-up protocol
}

\author{
Louis Leveuf ${ }^{1,2, *}$, Yann Marco ${ }^{1}$, Vincent Le Saux ${ }^{1}$, Libor Navratil ${ }^{1}$, Sylvain Leclercq ${ }^{3}$, and Jérôme Olhagaray ${ }^{2}$ \\ ${ }^{1}$ ENSTA Bretagne - iRDL, UMR CNRS 6027, 29200 Brest, France \\ ${ }^{2}$ Safran Composites, 91760 Itteville, France \\ ${ }^{3}$ Safran Landing Systems, 78140 Vélizy-Villacoublay, France
}

\begin{abstract}
This study deals with the characterization of the fatigue lifetime of a short carbon fiber reinforced PEEK matrix thermoplastic composite using a heat build-up protocol. Several commercial grades and fillers ratios are considered in order to challenge the ability of the technique to capture the influence of these variations. First, the materials investigated and the experimental protocols are described. The way to build the heat build-up curves is outlined. Then the results obtained from the heat build-up and the fatigue experiments are presented. Finally these results are used to discuss two main points. The first one is the validity of the hypotheses needed to apply the approach, ranging from the evaluation of the dissipation to the response during the fatigue tests. The second one is the capability to predict the fatigue curves accurately throughout an energetic criterion and to catch the influence of the variation of material on the fatigue properties. The results presented here are published with more details in [47].
\end{abstract}

\section{Introduction}

Short-fiber-reinforced thermoplastics (SFRT) provide a major opportunity today to obtain lightweight parts at a reasonable cost and have been widely investigated [1-6]. Over the last few years, this kind of materials has been used for structural components in the automotive industry to replace die casting aluminum or stamped steel parts [7-11]. The possible use of these materials for aeronautic applications is also under consideration. The main reasons are the reasonable properties of these materials, especially when using a thermostable matrix, and the use of the injection molding process which allows both a cost reduction and the definition of complex parts with stiffeners and ribs, to circumvent the loss in rigidity compared to metallic solutions. Fatigue campaigns are usually very time consuming, especially for polymeric materials as the test frequency cannot be very high to avoid any bias brought about by the heat build-up induced under cyclic solicitations. As these materials also provide a high versatility in fiber content, grades and suppliers, a screening tool, able to discriminate good candidates for fatigue applications quickly, prior to a full fatigue campaign, would be a crucial asset. One of the experimental tools to do so could be based on the analysis of the heat build-up response. Since the pioneer studies [12, 13], this technique has become of considerable interest over the last few years, on metallic materials for empirical approaches [14-17] or throughout constitutive modeling [18-20]. This is explained mainly by the considerable development of thermography devices and analysis [16, 21, and 22]. The extension of the technique to other materials has also been tested [17] and is now applied, following different approaches, to rubbers [23- 25], continuous fiber composites [26, 27] and short fiber reinforced plastics $[11,28,29]$. All these approaches use the evolution of the sample temperature but can be quite different. Some of them rely on the temperature only, which is quite limited as temperature is dependent on the frequency, on the samples' geometry and on the thermal boundary conditions. These analyses therefore give no access to an intrinsic parameter. Most of the protocols now seek the dissipated energy associated with the temperature variations. This evaluation is not trivial and requires solving the heat equation according to a wide range of hypotheses both on the temporal aspects (adiabatic or stabilized configurations) and on the spatial distribution of the dissipation sources. This evolution of the dissipated energy versus the amplitude of solicitation (strain or stress, depending on the materials tested) is then generated and is called the heat build-up curve. The following step is to relate it to the "fatigue properties", which could cover a pretty wide range of ambitions. The most basic is to evaluate an unlimited fatigue lifetime or at least a lifetime high enough compared to the range of cycles aimed at by the application. This analysis uses the change of shape of the heat build-up curve to identify this threshold. A more interesting objective is to predict the fatigue mean curve. This approach relies on the use of an energy based fatigue criterion and could be determined either empirically [23, 25, 29], with the help 
of a constitutive model $[18,30]$, or using additional microstructural measurements $[31,32]$. In all cases, it is necessary to check if the method can be applied, because a main difference of this approach compared to classic fatigue tests is that it relates the response over a few initial cycles to the fatigue failure occurring after several days or weeks of testing. It is therefore necessary to check that the constitutive response under fatigue conditions reaches a stationary state within this limited number of cycles [33, 34], before relating the cyclic thermomechanical response under several loading amplitudes to the fatigue lifetime. An even more desirable objective is to predict the full fatigue curve including the fatigue scattering from the heat build-up curve. This has been proven to be possible on several metallic materials [18-20], by relating the dissipated energy to the damage mechanisms throughout a constitutive modeling including a probabilistic failure criterion. This last approach is clearly the most appealing but it faces several difficulties for short fiber reinforced composites. The main difficulty is the extremely numerous dissipation phenomena possibly involved in the material response under fatigue loading, ranging from visco-elasticity to damage and plasticity [35-38]. Despite numerous studies $[6,39,40]$, relating the macroscopic dissipation to the basic fatigue mechanisms remains an open issue. A second issue is that predicting a relevant local dissipation and thus reliable fatigue predictions requires a very accurate evaluation of the hysteretic loop, which remains clearly difficult for these materials [41, 42, 46]. A last point is that a rich modeling approach usually also requires a rich testing campaign, involving a long preliminary study for each compound tested. This could not be integrated easily in a fast testing approach.

This study focuses on a thermostable matrix reinforced with short carbon fibers and aims at three main objectives. The first is to check if the hypotheses required to apply the approach is valid for these materials, both from a measurement point of view (adiabaticity, spatial distribution of the dissipation sources) and from a criterion point of view (evaluation possible over a limited amount of cycles). The second is to check the accuracy of the technique to predict the mean fatigue curve of these materials. The last is to challenge its capacity to be used as a fast screening tool to discriminate quickly several possible materials, for different grades, suppliers and carbon fiber amount. This study provides two main original aspects compared to the team's previous studies. The first one is the application of the heat build-up approach to these materials that have not been investigated yet. The second is the use of a specific identification of the temperature variation giving access to both dissipation and thermoelastic couplings, for each pixel of the measured area.

A first section presents the material investigated and the samples' geometry. In the second section, the experimental protocols used for the fatigue tests, the thermal characterization and the determination of the heat build-up curve are presented. The third section presents the results obtained both for the fatigue and the heat build-up tests. The fourth section provides an analysis of the results obtained and discusses the objectives of the paper, i.e. the consistency of the hypotheses and the capacity of the approach to predict the fatigue properties accurately and to discriminate quickly the fatigue properties of several close materials.

\section{Experimental settings}

\subsection{Materials and samples}

Four different materials were tested in this study, with different kinds of polymer matrix and amounts of carbon fibers (referenced as A, B, C, D). All the polymer matrices were thermostable materials sourced from several suppliers and are either PEEK or PAEK matrix reinforced with $30 \%$ to $40 \%$ (in weight) of short carbon fibers. It is important to underline that all these materials are close and are potential candidates for industrial applications.

Two different geometries were tested in this study. Figure 1 presents the shape and the geometrical parameters and Table 1 provides the parameters for the two different geometries. These samples were milled from a rectangular plate (nominal dimensions $250 \mathrm{~mm}$, $100 \mathrm{~mm}$ ), injection molded through a film gate on the shorter edge. The longitudinal axis of the samples was the main flow direction. Table 2 presents an overview of the materials tested and of the samples considered.

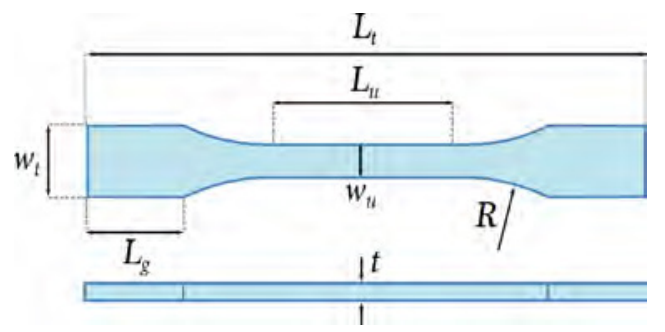

Fig. 1. Sample geometry. The values of the parameters are given in Table 1.

Table 1. Dimensions of the specimens (in $\mathrm{mm}$ ).

\begin{tabular}{c|c|c}
\hline Parameter & Type 1 & Type 2 \\
\hline $\boldsymbol{L}_{\boldsymbol{t}}$ & 170 & 99 \\
$\boldsymbol{L}_{\boldsymbol{u}}$ & 50 & 29 \\
$\boldsymbol{L}_{\boldsymbol{g}}$ & 30 & 18.75 \\
$\mathbf{t}$ & 4 & 3.25 \\
$\boldsymbol{w}_{\boldsymbol{t}}$ & 20 & 18 \\
$\boldsymbol{w}_{\boldsymbol{u}}$ & 10 & 11.9 \\
$\mathbf{R}$ & 60 & 45
\end{tabular}




\subsection{Fatigue tests}

The tests were performed on an INSTRON servohydraulic testing machine (capacity of $100 \mathrm{kN}$ ). The tests were force controlled, the load ratio was set to $\mathrm{R}=0$ and the frequency was $2 \mathrm{~Hz}$. All the tests were performed at room temperature $\left(25^{\circ} \mathrm{C}\right)$. The number of cycles between the initiation and the final failure was less than $3 \%$ of the total lifetime. To simplify the analysis, the number of cycles to initiation was therefore considered equal to that leading to the final failure. For each test, an evaluation of the dissipated energy according to the protocol described in Section 2.4.2 was achieved. For confidentiality issues, all the energetic and mechanical data were divided by arbitrary values, kept the same for all materials to allow comparison.

Table 2. References of the materials tested

\begin{tabular}{c|c|c|c}
\hline Reference & $\begin{array}{c}\text { Polymer } \\
\text { Matrix }\end{array}$ & $\begin{array}{c}\text { Carbon } \\
\text { fibers } \\
\text { (\%w) }\end{array}$ & Geometry \\
\hline $\boldsymbol{A}_{\mathbf{1}}$ & Grade 1 & 30 & Type 1 \\
$\boldsymbol{A}_{\mathbf{2}}$ & Grade 1 & 30 & Type 2 \\
$\boldsymbol{B}$ & Grade 2 & 30 & Type 2 \\
$\mathbf{C}$ & Grade 3 & 30 & Type 2 \\
$\boldsymbol{D}$ & Grade 2 & 40 & Type 2
\end{tabular}

\subsection{Thermal device}

The thermal acquisition was performed using a FLIR SC7600-BB infrared camera. The device is equipped with a Stirling-cycle cooled Indium-Antimonide (InSb) Focal Plane Array (FPA). The FPA is a $512 \times 640$ array of detectors digitized on 14 bits and sensitive in the 1.5$5.1 \mu \mathrm{m}$ spectral band with a $15 \mu \mathrm{m}$ pitch (distance between two detectors). The focal length of the optical lens is $50 \mathrm{~mm}$, and its transmissivity is 0.94 . For all the experiments, the integration time was set to $1800 \mu \mathrm{s}$. The chosen thermal acquisition frequency was $30 \mathrm{~Hz}$. In order to improve the thermal resolution (compared to classic thermal calibration), a compensated pixelwise calibration was used [22]. After this specific calibration, a NETD (Noise Equivalent Temperature Difference) of $8 \mathrm{mK}$ was obtained for differential measurements.

\subsection{Generation of the heat build-up curves}

\subsubsection{Heat build-up protocol}

All the tests were carried out at room temperature $\left(25^{\circ} \mathrm{C}\right)$. Special attention was paid to the experimental conditions in order to avoid the influence of the environment on the measurements as much as possible. The sample was painted with high emissivity paint (measured value of 0.95 ), surrounded by a black box in order to limit any reflections and a dark sheet covered the experimental set-up.

The principle of a heat build-up protocol basically consists in submitting a sample to a succession of cyclic tests of increasing stress amplitude, and to record the evolution of the temperature for each sequence. The analysis of the dissipated energy from the thermal resolution requires the heat equation to be resolved, as presented in Section 2.4.2. This analysis can be based either on a stationary state or on an adiabatic hypothesis $[17,25,43]$. The choice of the mechanical protocol depends of course on the chosen analysis.

In this study, the adiabatic analysis was selected, which led to a mechanical protocol focused on the first loading cycles. Each loading block was composed of two stages. The first was the loading step: the sample was submitted to 30 cycles according to the stress amplitude considered in the block. The tests were force controlled, the load ratio was set to $\mathrm{R}=0$ and the frequency was $2 \mathrm{~Hz}$. During this stage, a thermal acquisition was performed, supplying the thermal images that would be analyzed to follow the temperature evolution and to determine the dissipated energy. The second stage was a cooling step lasting between 3 to 15 minutes, in order to let the sample cool down to the ambient temperature and to have comparable initial conditions from one loading block to another. This mechanical protocol was repeated for each block, and allowed the dissipated energy to relate to the force amplitude. The last block ran until the failure of the sample.

\subsubsection{Thermal measurements and determination of the cyclic dissipated energy}

The experimental determination of the dissipated energy fields relies on the solving of the heat equation [21, 44]. Under some classic assumptions (including adiabaticity) detailed in former publications [11, 25, 43, 44], the heat equation can be written as

$$
\left.\rho c_{p} \frac{\partial \theta}{\partial t}\right|_{t \rightarrow 0}=f_{r} \Delta^{\star}+\mathcal{C}_{e}
$$

With $\theta$ the temperature variation, $f_{r}$ the mechanical frequency, $\Delta^{*}$ the cyclic dissipated energy and $\mathcal{C}_{e}$ the thermo-elastic coupling contribution, while $\rho$ and $c_{p}$ stand for the specific mass and specific heat, respectively. Under sinusoidal cyclic loading, the solution of this equation writes [16]

$$
\forall t \in \mathbb{R}^{+}, \theta(t)=\frac{f_{r}}{\rho c_{p}} \Delta^{\star} t+c_{e} \sin \left(2 \pi f_{r} t+\phi\right)
$$

This equation is local and the rigorous identification of the cyclic dissipation $\Delta^{*}$ and elastic coupling $\mathcal{C}_{e}$ is not straightforward because the sample is moving whereas the camera is fixed. It is therefore necessary to compensate the displacement of the sample and to transpose the fields measured into the reference configuration before performing the temporal analysis. To do so, the displacement of a high emissivity marker printed on the sample is analyzed using a home developed software Belenos [46]. The thermal fields are 
then transposed in the reference configuration prior to any other analysis.

Figure 2 presents the typical evolution of the temperature during one block (circular plain markers), obtained for one pixel of the observed zone. Two main classic phenomena are clearly observed here. The first is a global rise in temperature, which is related to dissipation. The second is the oscillation related to the thermo-elastic coupling. This signal is used to identify the dissipation and the amplitude of the thermo-elastic coupling, by fitting the unknowns of Equation 2 (i.e. $\Delta^{\star}$ and $c_{e}$ ). This identification is illustrated in Figure 2 (continuous line). As highlighted by that figure, the next question is the choice of the temporal window used to perform the identification. In order to make this choice, the respect of two hypotheses has to be considered. The first is that the cyclic dissipation is constant from one cycle to another. For the materials studying the mechanical hysteresis is stabilized within 4 cycles [47]. The temporal window should therefore start ideally after 4 cycles (i.e. 2 seconds). The second hypothesis to be checked is the adiabatic condition, i.e. that the time considered to perform the evaluation is far below the characteristic time allowing for thermal exchanges. In the case considered here, this characteristic time (evaluated from the temperature drop during the cooling steps was around 300s). A temporal window within the 5 first seconds from the start of the experiment seemed reasonable. The identification was therefore performed over a temporal window of 3 seconds after the 4 th cycle, as illustrated in Figure 2. One can see that the function identified also fitted the experimental signal very well over the full duration of the loading step, which validates the adiabaticity hypothesis.

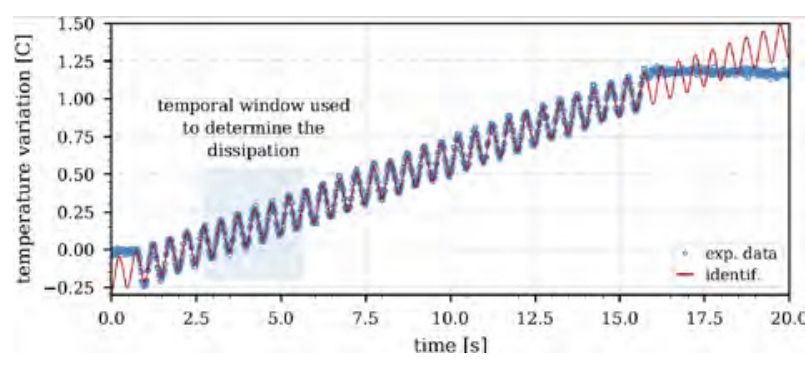

Fig. 2. Typical thermal measurements during one block of the heat build-up protocol.

This identification performed for every pixel of the observed zone, leading to the generation of the fields of dissipation and thermo-elastic coupling, identified over the temporal window. As explained in Section 2.4.1, several loading blocks with increasing force amplitudes were performed. For each loading block, the fields of dissipated energy and thermo-elastic coupling were evaluated. As illustrated Figure 3, the mean dissipation was computed over a given area, which led to the generation of the full heat build-up curve. As the dissipation field is homogenous over the gauge length of the sample, this evaluation is not dependant on the size of the averaging area.

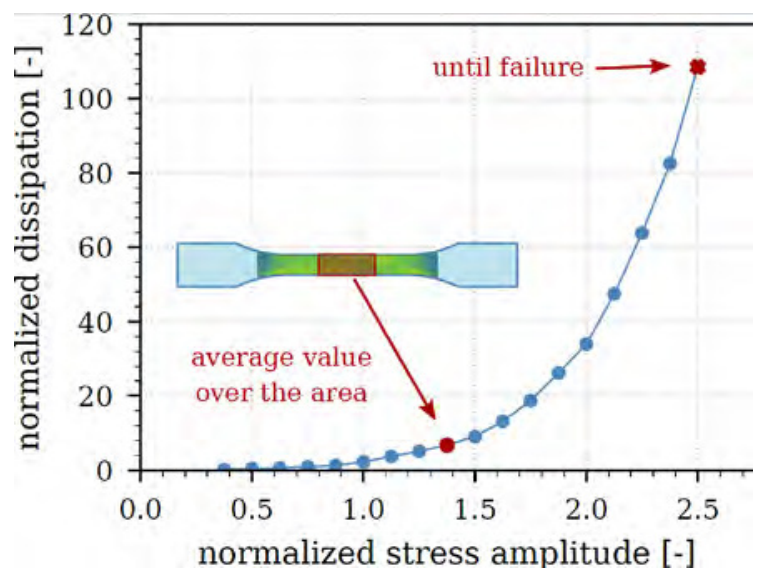

Fig. 3. Heat build-up curve relating the dissipated energy per cycle (normalized) to the maximum nominal principal strain (normalized). Curve associated to the material A2.

\section{Results}

\subsection{Fatigue tests}

The evolutions of the cyclic mechanical features were recorded during the fatigue tests. The secant modulus, the hysteretic loop and the residual strain (i.e. the strain at the end of each cycle) were computed from the nominal strain and stress derived from the extensometer and the load cell. These evolutions illustrate that the hysteresis remained stable over the full fatigue test and that the value evaluated at the beginning of the fatigue test was relevant up to the fatigue failure [47]. The evolutions of the secant modulus and of the residual strain were limited [47] for these materials compared to other thermoplastics $[3,29]$. The initial evolution seems consistent with the amount of cycles needed to reach a stabilized temperature.

\subsection{Fatigue curves}

In order to keep a reasonable amount of figures, the fatigue results are plotted in the Figures 7 and 8 comparing the criterion prediction to the experimental data. At this stage, only the experimental data are of interest. First of all, it is important to underline that these curves can obviously not be used for fatigue design as the amount of samples is too limited to afford a statistical approach. The goal of the fatigue test was only to provide a few failure data to check the tendencies predicted by the heat build-up predictions. The curve obtained for the material A with the sample geometry of type 1 nevertheless provides a richer database and it seems that the scattering remained limited. This also seems to be confirmed by the reasonable alignment obtained for the other materials and sample types. As mentioned previously, the dissipated energy (cf. Section 2) was measured. This allows both the classic S-N curves and a fatigue curve based on dissipated energy to be plotted. 


\subsection{Heat build-up curves}

The repeatability of the tests was systematically checked. Figure 4 illustrates the very good match between two different tests. It is also important to underline that these two heat build-up curves were generated throughout two different sequences of loading (different amount and amplitudes of loading blocks). This shows that there was no dependency of the heat build-up curve on the loading history. This is furthermore confirmed by the observation that the dissipated energy measured at the beginning of the fatigue tests (i.e. for one single block), was consistent with the heat build-up curve. This is also illustrated in Figure 4.

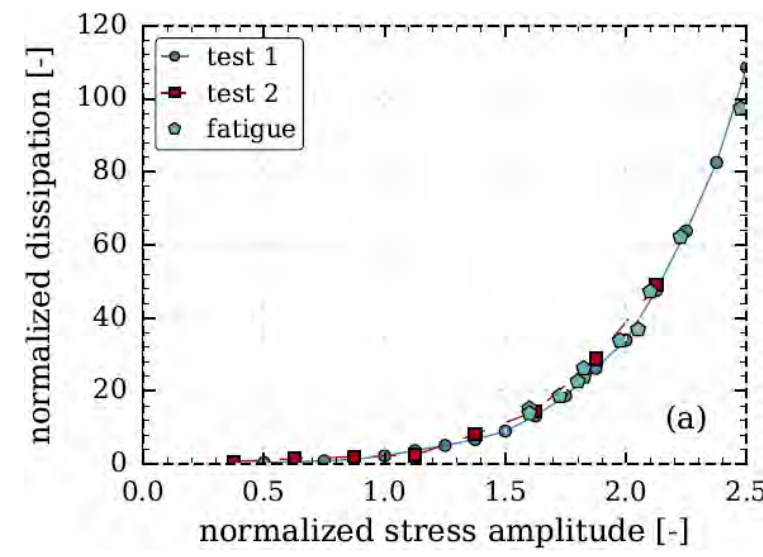

Fig. 4. Effect of the experimental scattering on the heat build-up curves. These data are obtained for the material A2.

The heat build-up curves obtained for the 4 materials studied are presented in Figure 5. One can observe that the dissipation responses can be markedly different, depending on the grade and the amount of fibers. One can also notice that the heat build-up curves obtained for the same materials, but using two different geometries (references $\mathrm{A}_{1}$ and $\mathrm{A}_{2}$ ), were different. This was due to their different thicknesses, leading to different microstructures.

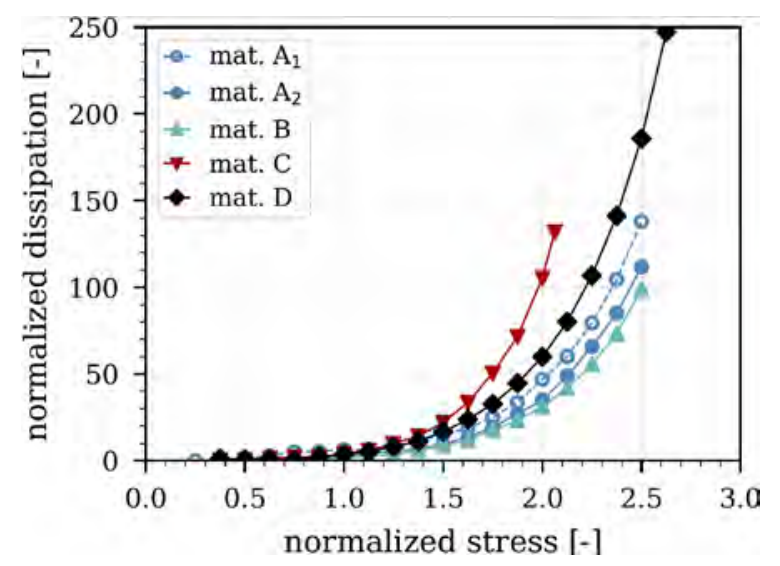

Fig. 5. Heat build-up curves for the studied materials.

\section{Analysis and discussion}

\subsection{Validation of the hypothesis for the heat build-up curves}

The objective of this paragraph is to sum-up and check the hypotheses required to deduce the fatigue properties from the heat build-up curve. A first set of hypotheses is related to the evaluation of the dissipated energy. In this case, a protocol valid for adiabatic conditions was applied, on the additional assumption of constant dissipation per cycle. Both conditions were validated in Section 2.4.2. Moreover, the energy evaluated seems representative of the whole section of the sample. Finally, the heat build-up test should be repeatable, which was checked for all the materials tested. A second set of hypotheses is related to the connection to the fatigue tests and the tentative prediction of the fatigue lifetime. First of all, the energy should be evaluated over the failure location, which is consistent here with a failure occurring in the gauge volume. Then, the dissipation evaluated for a fatigue test should be consistent with that obtained for the heat build-up test. That was checked in Section 2.4.2. Finally, the energy evaluated over a limited amount of cycles should be consistent with that dissipated during the fatigue test, which was checked in Section 2.4.2.

\subsection{Identification of the dissipated energy criterion parameters}

The heat build-up curve provides a relation between the stress amplitude and the dissipated energy. The next step is to use an energy based criterion to relate the dissipated energy to the fatigue lifetime. In former studies $[23,25$, 28,29 ], protocols using thermal measurements to predict the fatigue life throughout an energy based criterion proved to afford a very efficient prediction of the deterministic Wöhler curve (one sample, within less than one day) and for various materials. This very efficient approach is based on thermal data only and uses a fatigue criterion relating the number of cycles $\mathrm{N}$ to initiation to the cyclic dissipated energy $\Delta^{\star}$ with two parameters $\mathrm{b}$ and $\mathrm{C}$ that need to be identified

$$
\Delta^{\star} N^{b}=C
$$

The approach considered in $[11,25]$ consists in identifying regimes on the heat build-up curves based on a graphical analysis of the curve. This approach is possible only with a large amount of experimental data and thus a high number of blocks. This is possible here, thanks to the evaluation on a few cycles, avoiding any damage accumulation during the heat build-up test. To highlight these regimes (see Figure 8a), we propose here a new approach based on the cyclic dissipation rate computed using finite difference according to

$$
\dot{\Delta}^{\star}\left(\sigma_{n}+\frac{1}{2}\left[\sigma_{n+1}-\sigma_{n}\right]\right)=\frac{\Delta_{n+1}^{\star}-\Delta_{n}^{\star}}{\sigma_{n+1}-\sigma_{n}}
$$


These variations are computed using two following points and are associated to the mean stress values related to these points. Figure 6(a) shows the evolution of the cyclic dissipation rate for the experiments presented in Figure 4. The regimes can then be identified easily.
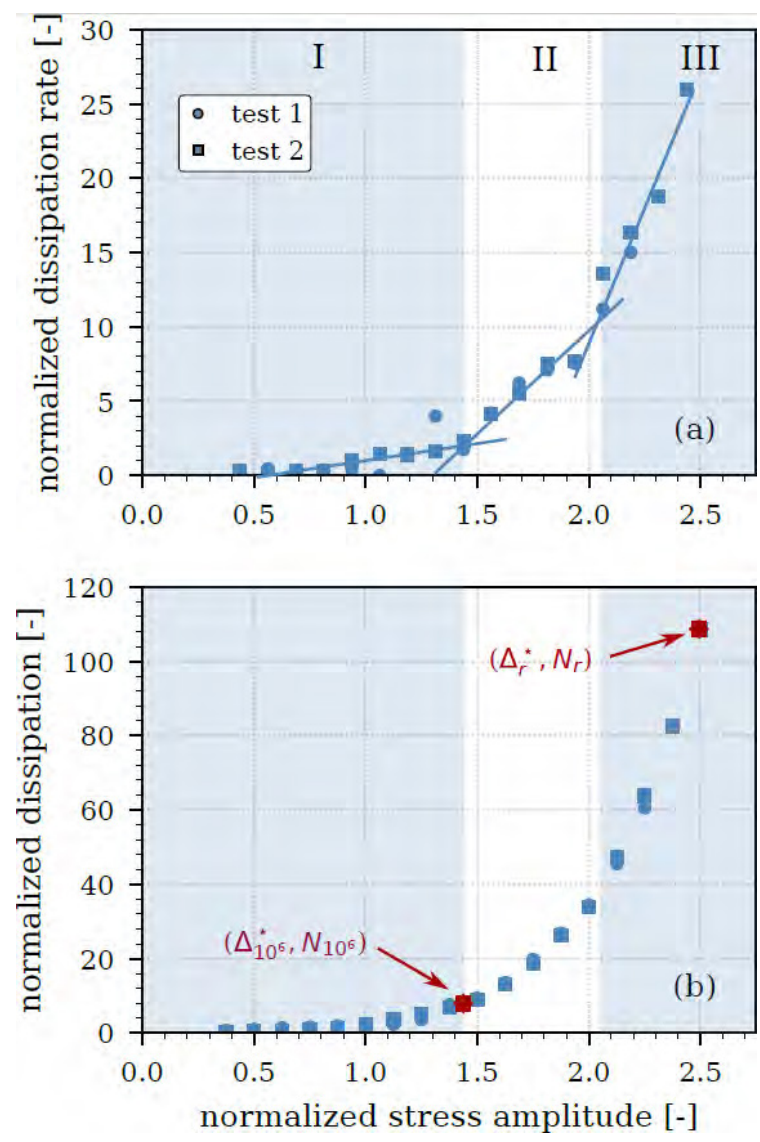

Fig. 6. Determination of the parameters based on a regimes approach for the material $\mathrm{A}_{2}$.

Two sets $\left(\Delta^{\star}, N\right)$ are needed to identify the parameters analytically. The first set $\left(\Delta_{R}^{\star}, N_{R}\right)$ was obtained from the last block that ran until the sample failure. The second set $\left(\Delta_{10^{6}}^{\star}, N_{10^{6}}\right)$ was obtained from the heat build-up curve at the transition between regimes I and II, as illustrated in Figure 6b. Here, the hypothesis is that the energy evaluated for this transition leads to a lifetime of $10^{6}$ cycles. This hypothesis is highly disputable as these materials do not show any clear fatigue limit. This point, initiated in other references [5, 44], deserves further discussion. In particular, the physical nature of the regimes identified is to be clarified as a clear split between the nonlinear effects of viscoelasticity, viscoplasticity and damage remains difficult for these materials [9, 38, and 42]. Modeling, of course, is fundamental in clarifying this point. A very recent paper [45] presents a comparison of the experimental curves to numerical simulations for one of the material investigated here. The model includes elasticity, plasticity and non-linear viscoelasticity coupled to plasticity and strain rate. The identification is achieved on a extensive database and validated throughout the heat buildup curve prediction. The agreement is good and it appears that the first regime can be related to pure visco-elastic effects, that the second one is correlated to the onset of plasticity even if the contribution remains limited to the overall dissipation and that the third regime is induced both by plastic effects and by the coupling of plasticity to non-linear visco-elasticity. In this study, the focus is on the application of the existing protocol and checking its relevancy for the materials tested. The identification procedure was applied to the 4 materials studied and the two samples' geometry (material A).

\subsection{Comparaison between fatigue curves and energetic predictions}

Figures 7 and 8 present the comparison between the experimental data obtained from the fatigue tests and the prediction of the dissipated energy criterion. Above, the fatigue curves are plotted using the dissipated energy as the fatigue indicator. Below, the classic S-N curve is plotted.
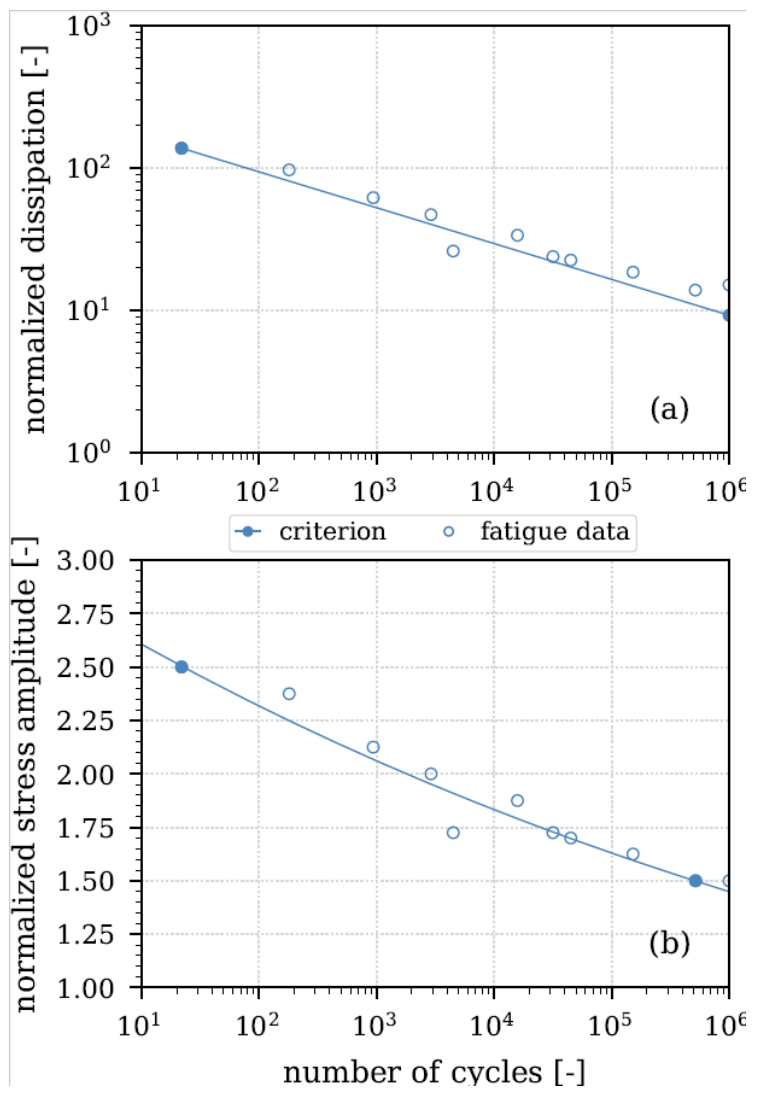

Fig. 7. Comparison between the fatigue data (empty markers) and the energetic criterion (line and filled markers) for the material $\mathrm{A}_{1}$ in terms of dissipation versus number of cycles (a) and nominal stress versus number of cycles (b).

In both cases, the correlation obtained is fairly good for all the materials and samples considered. It is important to underline that different heat build-up curves could nevertheless lead to similar S-N curves. A very 
interesting result is that, for the same material, different fatigue properties can be found due to different microstructures induced by injection. In any case, the technique manages to describe well the fatigue resistance of the materials investigated. It therefore appears to be a relevant tool to evaluate the best candidates for fatigue applications, prior to generating full fatigue curves using classic fatigue campaigns.
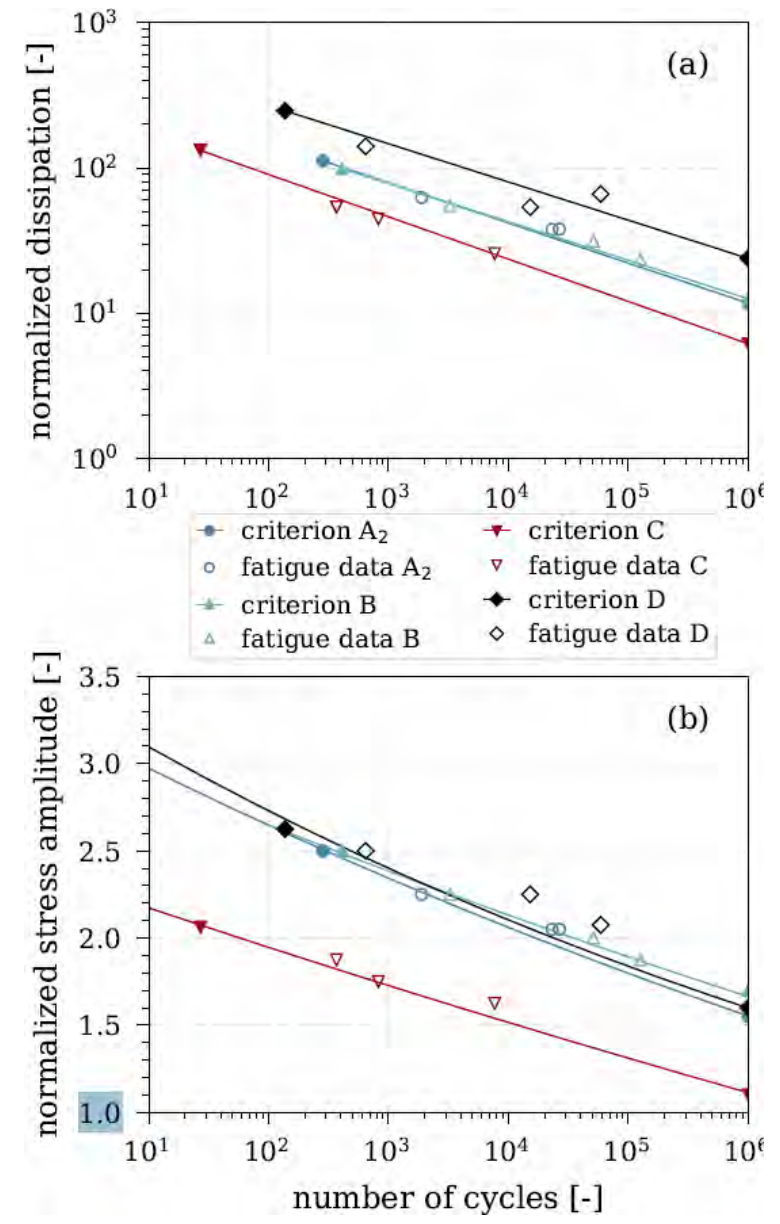

Fig. 8. Comparison between the fatigue data (empty markers) and the energetic criterion (line and filled markers) for the type 2 specimen in terms of dissipation versus number of cycles (a) and nominal stress versus number of cycles (b).

\section{Conclusions}

This paper focused on thermostable matrices, reinforced with short carbon fibers and aimed at three main objectives. The first was to highlight and investigate if a heat build-up approach can be applied to these materials. This was checked both from a measurement point of view (adiabaticity, spatial distribution of the dissipation sources) and from a criterion point of view (evaluation possible over a limited amount of cycles). Compared to previous papers on other materials published by the authors, the adiabatic analysis was here performed over each pixel, throughout the analysis of the temporal variation. A second objective was to check the ability of the technique to predict the mean fatigue curve of these materials. This was assessed on a wide database for one material and one sample geometry. The last objective was to challenge the capacity of the heat build-up technique to be used as a fast screening tool, i.e. to discriminate several possible materials quickly, for different grades, suppliers and carbon fiber amounts. The results obtained on 4 materials illustrated that the heat build-up protocol, defined and applied in this study, managed to predict the fatigue resistance of the materials investigated well, with one sample and within one day per material tested. It therefore appears to be a relevant tool to select the best candidates for fatigue applications from a given panel, prior to generating full fatigue curves using classic fatigue campaigns.

\section{References}

1. A. Zago, G. Springer, Fatigue lives of short _ber reinforced thermoplastics parts, Journal of Reinforced Plastics and Composites 20 (2001) 606-620.

2. C. Sonsino, E. Moosbrugger, Fatigue design of highly loaded short-glass-fibre reinforced polyamide parts in engine compartments, International Journal of Fatigue 30 (2008) 1279-1288.

3. Klimkeit, B., Etude expérimentale et modélisation du comportement en fatigue multiaxiale d'un polymère renforcé pour application automobile, Ph.D. thesis, ENSMA (2014).

4. A. Bernasconi, P. Davoli, C. Armanni, Fatigue strength of a clutch pedal made of reprocessed short glass fibre reinforced polyamide, International Journal of Fatigue 32 (2010) 100-107. 5. Serrano, L., Thermomechanical characterization of the fatigue behaviour of short fibers reinforced thermoplastic, Ph.D. thesis, Université de Bretagne Occidentale (2015).

6. S. Mortazavian, A. Fatemi, Fatigue behavior and modeling of short fiber reinforced polymer composites: A literature review, International Journal of Fatigue 70 (2015) 297-321.

7. M. De Monte, E. Moosbrugger, M. Quaresimin, Inuence of temperature and thickness on the off-axis behaviour of short glass fibre reinforced polyamide 6.6 - quasi-static loading, Composites Part A: Applied Science and Manufacturing 38 (2010) 859-871.

8. B. Klimkeit, Y. Nadot, S. Castagnet, C. Nadot-Martin, C. Dumas, S. Bergamo, C. Sonsino, A. Buter, Multiaxial fatigue life assessment for reinforced polymers, International Journal of Fatigue 33 (2011) 766-780.

9. A. Launay, M. Maitournam, Y. Marco, I. Raoult, Multiaxial fatigue models for short glass fiber reinforced polyamide. Part II: fatigue life estimation, International Journal of Fatigue 47 (2013) 390-406.

10. A. Launay, Y. Marco, M. Maitournam, I. Raoult, Modelling the inuence of temperature and relative humidity on the time-dependent mechanical behaviour of a short glass fibre reinforced polyamide, Mechanics of Materials 56 (2013) 1-10. 11. L. Serrano, Y. Marco, V. Le Saux, G. Robert, P. Charrier, Fast prediction of the fatigue behavior of short-fiber-reinforced thermoplastics based on heat build-up measurements: application to heterogeneous cases, Continuum Mechanics and Thermodynamics 29 (2017) 1113-1133.

12. C. Stromeyer, The determination of fatigue limits under alternating stress conditions, Proceedings of the Royal Society of London. Series A. 90 (1914) 411-425.

13. H. Moore, J. Kommers, Fatigue of metals under repeated stress, Chemical and Metallurgical Engineering 25 (1921) 1141-1144. 
14. A. Galtier, Contribution à l'étude de l'endommagement des aciers sous sollicitations uni ou multiaxiales, Ph.D. thesis, ENSAM (1993).

15. M. Luong, Fatigue limit evaluation of metals using an infrared thermographic technique, Mechanics of Materials 28 (1998) 155-163.

16. J. Krapez, D. Pacou, G. Gardette, Lock-in thermography and fatigue limit of metals, in: Proceedings of QIRT'2000, Reims, 2000, pp. 277-282.

17. G. La Rosa, A. Risitano, Thermographic methodology for rapid determination of the fatigue limit of materials and mechanical components, International Journal of Fatigue 22 (2001) 65-73.

18. C. Doudard, S. Calloch, F. Hild, P. Cugy, A. Galtier, Identification of scatter in high cycle fatigue from temperature measurements, Comptes Rendus M_ecanique 10 (2004) 795801.

19. A. Ezanno, C. Doudard, S. Calloch, J. Heuzé, A new approach to characterizing and modeling the high cycle fatigue properties of cast materials based on self-heating measurements under cyclic loadings, International Journal of Fatigue 47 (2004) 232-243.

20. R. Munier, C. Doudard, S. Calloch, B. Weber, Determination of high cycle fatigue properties of a wide range of steel sheet grades from self-heating measurements, International Journal of Fatigue 63 (2014) 46-61.

21. A. Chrysochoos, H. Louche, An infrared image processing to analyse the calori_c e_ects accompanying strain localisation, International Journal of Engineering Science 38 (2000) 17591788 .

22. V. Le Saux, C. Doudard, Proposition of a compensated pixelwise calibration for photonic infrared cameras and comparison to classic calibration procedures: case of thermoelastic stress analysis, Infrared Physics and Technology 80 (2017) 83-92.

23. V. Le Saux, Y. Marco, C. Doudard, S. Calloch, P. Charrier, Fast evaluation of the fatigue lifetime of rubber-like materials based on a heat build-up protocol and micro-tomography measurements, International Journal of Fatigue 32 (2010) 1582-1590.

24. C. Cruanes, G. Berton, F. Lacroix, S. Meo, N. Ranganathan, Study of the fatigue behavior of the chloroprene rubber for uniaxial tests with infrared method, Elastomery 18 (2014) 3-9.

25. Y. Marco, I. Masquelier, V. Le Saux, P. Charrier, Fast prediction of the $\mathrm{W} \square$ ohler curve from thermal measurements for a wide range of NR and SBR compounds, Rubber Chemistry and Technology 90 (2017) 487-507.

26. T. L., M. Karama, B. Lorrain, Damage evolution and infrared thermography in woven composite laminates under fatigue loading, International Journal of Fatigue 28 (2006) 1867-1872.

27. M. Liakat, K. Khonsari, Analysis and life prediction of a composite laminate under cyclic loading, Composites Part B: Engineering 84 (2016) 98-108.

208. G. Meneghetti, M. Quaresimin, Fatigue strength assessment of a short fiber composite based on the specific heat dissipation, Composites Part B: Engineering 42 (2011) 217225.

29. L. Jégou, Y. Marco, V. Le Saux, S. Calloch, Fast prediction of the wöhler curve from heat build-up measurements on short fiber reinforced thermoplastics, International Journal of Fatigue 47 (2012) 259-267.

30. M. Poncelet, C. Doudard, S. Calloch, B. Weber, F. Hild, Probabilistic multiscale models and measurements of selfheating under multiaxial high cycle fatigue, Journal of the Mechanics and Physics of Solids 58 (2010) 578-593.

31. V. Le Saux, Y. Marco, S. Calloch, P. Charrier, Evaluation of the fatigue defect population in an elastomer using $\mathrm{x}$-ray computed micro-tomography, Polymer Engineering and Science 51 (2011) 1253-1263.

32. Y. Marco, B. Huneau, I. Masquelier, V. Le Saux, P. Charrier, Prediction of fatigue properties of natural rubber based on the descriptions of the cracks population and of the dissipated energy, Polymer Testing 59 (2017) 67-74.

33. A. Constantinescu, K. Dang Van, M. Maitournam, A unified approach for high and low cycle fatigue based on shakedown concepts, Fatigue and Fracture of Engineering Materials and Structures 26 (2003) 1065-1077.

34. A. Benoit, M. Maitournam, L. Rémy, F. Oger, Cyclic behaviour of structures under thermomechanical loadings: Application to exhaust manifolds, International Journal of Fatigue 38 (2012) 65-74.

35. A. Drozdov, A. Al-Mulla, R. Gupta, Finite viscoplasticity of polycarbonate reinforced with short glass fibers, Mechanics of Materials 37 (2005) 473-491.

36. H. Nouri, F. Meraghni, P. Lory, Fatigue damage model for injection-molded short glass fibre reinforced thermoplastics, International Journal of Fatigue 31 (2009) 934-942.

37. A. Andriyana, N. Billon, L. Silva, Mechanical response of a short fiber-reinforced thermoplastic: experimental investigation and continuum mechanical loading, European Journal of Mechanics A 29 (2010) 1065-1077.

38. A. Launay, M. Maitournam, Y. Marco, I. Raoult, F. Szmytka, Cyclic behaviour of short glass fibre reinforced polyamide: experimental study and constitutive equations, International Journal of Plasticity 27 (2011) 1267-1293.

39. M. Arif, F. Meraghni, N. Chemisky, Y. ad Despringre, G. Robert, In situ damage mechanisms investigation of PA66/GF30 composite: effect of relative humidity, Composites Part B: Engineering 58 (2014) 487-495.

40. H. Rolland, N. Saintier, P. Wilson, J. Merzeau, G. Robert, In situ X-ray tomography investigation on damage mechanisms in short glass fibre reinforced thermoplastics: Effects of fibre orientation and relative humidity, Composites Part B: Engineering 109 (2017) 1359-1368.

41. A. Launay, M. Maitournam, Y. Marco, I. Raoult, Multiaxial fatigue models for short glass fiber reinforced polyamide. Part I: nonlinear anisotropic constitutive behavior for cyclic response, International Journal of Fatigue 47 (2013) 382-389.

42. A. Krairi, I. Doghri, A thermodynamically-based constitutive model for thermoplastic polymers coupling viscoelasticity, viscoplasticity and ductile damage, International Journal of Plasticity 60 (2014) 163-181.

43. I. Masquelier, Y. Marco, V. Le Saux, S. Calloch, P. Charrier, Determination of dissipated energy fields from temperature mappings on a rubber-like structural sample: Experiments and comparison to numerical simulations, Mechanics of Materials 80 (2015) 113-123.

44. Y. Marco, V. Le Saux, L. J_egou, A. Launay, L. Serrano, I. Raoult, S. Calloch, Dissipation analysis in SFRP structural samples: thermomechanical analysis and comparison to numerical simulations, International Journal of Fatigue 67 (2014) 142-150.

45. V. Le Saux, Belenos users manual, Internal report. ENSTA Bretagne (2017).

46. L. Leveuf, L. Navratil, V. Le Saux, Y. Marco, J. Olhagaray, S. Leclercq, Constitutive equations for the cyclic behaviour of short carbon fibre-reinforced thermoplastics and identification on a uniaxial database, Continuum Mechanics and Thermodynamics accepted (2018) 1-20.

47. L. Leveuf, Y. Marco, V. Le Saux, J. Olhagaray, S. Leclercq, Fast screening of the fatigue properties of thermoplastics reinforced with short carbon fibers based on thermal measurements, Submitted to Polymer Testing (January, 2018) 